\title{
Produção de uma cartilha educativa sobre a prevenção e controle da hipertensão arterial
}

\section{Production of an educational booklet on the prevention and control of arterial hypertension}

Alcínia Braga de Lima Arruda ${ }^{1 *}$, Hoberlania Vieira Alves Oliveira² ${ }^{2}$ Adriano Evangelista Maia $^{2}$, Isabelle de Fátima Vieira Camelo Maia², Emanuela Kelly Silva de Sousa², Josyelle Moreira de Sousa ${ }^{2}$, Arthur Chagas de Sousa ${ }^{2}$, Luis Ednilson Maciel Gonzaga ${ }^{2}$

\begin{abstract}
RESUMO
O objetivo deste trabalho foi descrever à elaboração de uma cartilha educativa para idosas. Trata-se de um estudo descritivo, do tipo relato de experiência, realizado nos meses de julho a agosto de 2021, o qual descreve o processo da produção do material educativo. A cartilha foi confeccionada acompanhando as seguintes etapas: definição do tema da cartilha, levantamento bibliográfico do assunto e a montagem da cartilha propriamente dita. Desse modo, foi possível colocar nesta cartilha informações importantes que permitem o reconhecimento da hipertensão arterial, seus sintomas, prevenção e controle. O resultado foi uma cartilha intitulada de "Prevenção e Controle da Hipertensão Arterial", composta por um total de 16

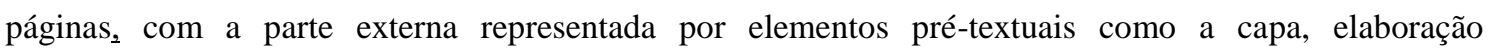
(identificação do autor do trabalho) e sumário; por elementos textuais representados pelo conteúdo, seguida pelos elementos pós-textuais, compostos pelas referências e por uma página de anotações do controle das medidas da pressão arterial da idosa. Na confecção da cartilha utilizou-se todas as sugestões sugeridas pela literatura especializada, conseguindo elaborar uma ferramenta didática simples e de fácil entendimento.
\end{abstract}

Palavras-chave: Promoção da saúde; Assistência integral à saúde; Materiais de Ensino.

\begin{abstract}
The objective of this work was to describe the elaboration of an educational booklet for the elderly. This is a descriptive study, of the experience report type, carried out from July to August 2021, which describes the process of production of educational material. The booklet was prepared following the following steps: definition of the theme of the booklet, bibliographic survey of the subject and the assembly of the booklet itself. In this way, it was possible to include in this booklet important information that allows the recognition of arterial hypertension, its symptoms, prevention and control. The result was a booklet entitled "Prevention and Control of Hypertension", comprising a total of 16 pages, with the outside represented by pre-textual elements such as the cover, elaboration (identification of the author of the work) and summary; by textual elements represented by the content, followed by post-textual elements, composed of references and a page of notes on the control of blood pressure measurements in the elderly. In the preparation of the booklet, all the suggestions suggested by the specialized literature were used, managing to develop a simple and easyto-understand teaching tool.
\end{abstract}

Keywords: Health promotion; Comprehensive health care; Teaching materials.

\footnotetext{
${ }^{1}$ Universidade Federal do Ceará.*E-mail: alcinialima@gmail.com

${ }^{2}$ Universidade Federal do Ceará
} 


\section{INTRODUÇÃO}

O envelhecimento humano é um fenômeno natural desejado por toda a sociedade, entretanto ele é um processo complexo, pois provoca sérias transformações biológicas, psicológicas e sociais nos longevos (BORGES et al., 2012; DANTAS; SANTOS, 2017).

As modificações biológicas dizem respeito às alterações anatômicas, fisiológicas e bioquímicas que ocorrem no organismo (BORGES et al., 2012; DANTAS; SANTOS, 2017). As transformações psicológicas se referem às mudanças do humor, raciocínio, concentração, memória e à adequação do indivíduo ao novo cotidiano da sociedade. Enquanto, que as modificações sociais são estabelecidas pelas mudanças do comportamento do indivíduo com o ambiente e/ou contexto sociocultural no qual está inserido (BORGES et al., 2012; DANTAS; SANTOS, 2017).

Dessa maneira, as transformações que ocorrem no indivíduo idoso provocam alterações nos padrões das doenças e mortalidade na terceira idade, ocorrendo predomínio das doenças crônicas não transmissíveis (DCNT) e redução dos óbitos ocasionadas pelas doenças infecciosas (PEREIRA et al., 2009).

As doenças crônicas não transmissíveis são aquelas que têm causas multifatoriais, possuem um curso clínico lento e de longa duração e os indivíduos portadores das DCNT necessitam de acompanhamento médico contínuo e realização de exames periódicos (PEREIRA et al., 2009; SBC, 2010; FERREIRA et al., 2012; VERAS; OLIVEIRA, 2018).

As DCNT são um dos maiores problemas de saúde pública da atualidade. Dados da literatura mostram que as doenças crônicas não transmissíveis são responsáveis por $70 \%$ das mortes ocorridas no mundo. Além de um alto número de óbitos, as DCNT elevam o número de internações, aumentam o uso de medicamentos, limitam as atividades dos idosos e causam um impacto econômico negativo para a sociedade (MOURA; CARVALHO; SILVA, 2007; MALTA et al., 2017).

Para o Ministério da Saúde (2009), o envelhecimento da população associado ao consumo do tabaco e bebida alcoólica, inatividadae física e à alimentação inadequada são os grandes responsáveis pelo aumento da incidência e prevalência das doenças crônicas não transmissíveis (DCNT). E, dentre as principais DCNT que acometem os longevos, destacam-se a Hipertensão Arterial, o Diabetes e as Doenças Respiratórias Crônicas (BRASIL, 2009). 
A hipertensão arterial sistêmica (HAS) é um problema grave de saúde pública, devido sua alta frequência e risco de mortalidade. Sua prevalência aumenta progressivamente com o envelhecimento, sendo a prevalência de HAS superior a $50 \%$ na faixa etária acima de 65 anos. No Brasil, dados da Pesquisa Nacioal de Saúde de 2013, mostraram que $21,4 \%$ dos adultos brasileiros se autodeclaram portadores de hipertensão (IBGE, 2013; SOUSA et al., 2019; BARROSO, 2021).

A prevenção da hipertensão arterial consiste em atividades estratégicas, desenvolvidas para prevenir ou controlar os fatores de risco responsáveis pela enfermidade. As mudanças dos hábitos alimentares, a realização da prática regular de atividades físicas, a diminuição do consumo do álcool e do uso do fumo e à adesão medicamentosa são atitudes que melhoram a qualidade de vida dos longevos hipertensos (SOUSA et al., 2019).

A carência de programas que atendam a população da terceira idade é real. Entretanto, sabe-se que práticas educativas para idosos, familiares, cuidadores e a comunidade em geral, podem transformar o processo saúde/doença, prevenindo ou retardando a ocorrência de enfermidades, diminuindo o uso indiscriminado de medicamentos e reduzindo as incapacidades físicas (VERAS, 2012).

No processo educativo as atividades de promoção em saúde podem ser realizadas de diversas maneiras. O educador pode atuar verbalmente, esclarecendo oralmente as dúvidas do paciente e seus familiars. O educador pode agir com a utilização da escrita, assim, as dúvidas e recomendações são redigidas ou impressas para não serem esquecidas. $\mathrm{Ou}$, educador pode atuar utilizando atividades gráficas, dessa maneira, as instruções são passadas para o pacientes através de desenhos, diagramas, ilustrações, figuras, textos ou materiais educativos impressos (DUTRA; COELHO, 2006; CASTRO; LIMA JÚNIOR, 2014).

Na realização de atividades gráficas, diversos recursos educativos podem ser usados para a divulgação do conhecimento, sendo os materiais escritos/impressos os mais utilizados para isso. E, dentre esses, merece destaque as cartilhas (CASTRO; LIMA JÚNIOR, 2014).

As cartilhas educativas são definidas como materiais impressos que têm a função de ensinar e reforçar as orientações verbalizadas, além de fornecer informações importantes acerca dos cuidados, fatores de riscos, prevenção e tratamento 
medicamentoso utilizados nas enfermidades (MOREIRA; NÓBREGA; SILVA, 2003; MENDONÇA, 2004; TORRES et al., 2009 CASTRO; LIMA JÚNIOR, 2014).

Existem diversos estudos que mostram o desenvolvimento desta tecnologia, bem como a sua importância para a sociedade. Dente as diversas cartilhas, podemos citar por exemplo: alimentação saudável durante a gravidez (OLIVEIRA; LOPES; FERNANDES, 2014); prevenção da transmissão vertical pelo HIV (LIMA, 2014); autocuidado com os pés de pessoas com diabetes (GALDINO, 2014); promoção do autocuidado na hanseníase (MARTINS et al., 2019); prevenção e controle da diabetes (LIMA, 2020); orientação de feirantes quanto à prevenção do novo coronavírus e da COVID-19 (AMORIM et al., 2020).

\section{OBJETIVO}

Diante de tais considerações, resolvemos realizar este trabalho com o objetivo de descrever a produção de uma cartilha educativa sobre a prevenção e controle da hipertensão arterial para ser utilizada com idosas residentes em uma Instituição de Longa Permanência (ILP).

\section{METODOLOGIA}

Trata-se de um estudo descritivo, do tipo relato de experiência, descrevendo as etapas envolvidas na produção de uma cartilha educativa sobre a prevenção e o controle da hipertensão arterial.

A partir da definição do tema da cartilha, ocorreu o levantamento bibliográfico sobre o assunto "prevenção e o controle da hipertensão arterial”, utilizando as bases de dados Literatura Latino-Americana e do Caribe em Ciência da Saúde (LILACS) e Scientific Electronic Library Online (SciELO) para a busca dos artigos científicos.

De posse dos artigos, foi realizada a produção da cartilha, quanto aos conteúdos pré-textual e textual, a linguagem, as ilustrações e layout. Nessa etapa ocorreu o agrupamento das informações com as ilustrações realizadas. Os programas utilizados para confecção das ilustrações foram o Corel Draw Essentials para desenhar e o Adobe Photoshop para colorir as figuras. E por fim, realizou-se a diagramação da cartilha e configuração das páginas por meio do programa de computação gráfica.

Por se tratar de um estudo metodológico de construção de cartilha, o presente estudo não precisou ser submetido à avaliação do Comitê de Ética em Pesquisa. 


\section{RESULTADOS E DISCUSSÃO}

A produção da cartilha educativa foi realizada de forma sequencial em três etapas: na primeira fase foi definido o tema a ser abordado no conteúdo textual; na segunda etapa foi realizado o levantamento do conteúdo/assunto e na terceira fase foi feita a montagem da cartilha.

a) Definição do assunto

A definição do conteúdo da cartilha surgiu da experiência dos autores como membros de um projeto de extensão com idosas residentes em uma Instituição de Longa Permanência (ILP). Os autores perceberam que muitas idosas tinham hipertensão arterial sistêmica (HAS), faziam uso de vários medicamentos e não tinham um material educativo direcionado a sua doença. Com isso, resolveu-se produzir uma cartilha sobre esse assunto, com o objetivo de aumentar o nível de informação das idosas, bem como melhorar a prevenção e controle da HAS.

GOZZO et al. (2012), recomendam que ao elaborar o material educativo, é relevante que se conheça a realidade e expectativas do público-alvo, pois o assunto abordado na cartilha deve ser um fator que motive o interesse pela leitura.

b) Levantamento bibliográfico

Depois de definido o tema a ser abordado na cartilha, foi realizada a seleção dos conteúdos que seriam utilizados na construção da cartilha. Essa seleção teve por finalidade embasar cientificamente o assunto que seria abordado no material educativo. Foi realizado um levantamento bibliográfico nas bases de dados LILACS e SciELO, utilizando os descritores "Idosos, Hipertensão, Doenças não transmissíveis, Materiais de Ensino, Educação em saúde e Promoção da Saúde”. Foram empregados os operadores booleanos $A N D$ e $O R$ para restringir a pesquisa bibliográfica. Os artigos encontrados foram selecionados, através da leitura dos seus resumos e aqueles que mais se adequavam ao assunto e que estavam disponíveis na íntegra foram utilizados.

LIMA (2014) descreveu no seu estudo que o levantamento bibliográfico é uma das etapas mais importantes na construção de um material educativo visto que, a fundamentação científica dá segurança ao leitor e facilita à aquisição do conhecimento.

c) Montagem da cartilha

Primeiramente, o conteúdo textual da cartilha foi organizado em capítulos, seguido da elaboração das ilustrações, layout e design da cartilha. 
A montagem da cartilha foi desenvolvida utilizando os critérios de Moreira e colaboradores (2003), os quais descrevem os aspectos relacionados à ilustração, layout e linguagem que o indivíduo deve considerar ao elaborar um material educativo impresso. Nesta produção usou-se o programa Corel Draw Essentials para desenhar/ilustrar, o programa Adobe Photoshop para colorir as figuras e os programas Word e Power Point para finalizar a montagem da cartilha.

O conteúdo textual foi abordado de forma que houvesse concordância entre o título da cartilha e o texto interno. Para tanto, foram redigidas algumas perguntas e respostas no intuito de proporcionar um dinamismo durante a leitura e envolver as idosas no contexto da conversa.

As respostas contidas na cartilha quanto aos questionamentos, objetivaram esclarecer as dúvidas com uma linguagem simples, visando uma compreensão clara para o entendimento das idosas, cuidadores e/ou familiares. As informações mais relevantes sobre o tema foram organizadas em tópicos, utilizando uma linguagem informal.

Para Echer (2005), em um material educativo deve-se evitar o uso de uma linguagem técnica, pois a cartilha é construída para orientar pacientes e familiares e esses devem entender o que está escrito.

Moreira e colaboradores (2003) e Reberte (2008) enfatizam que é importante evitar o uso de termos técnicos e científicos, abreviaturas e siglas na dissertação de um material educativo, pois podem provocar interpretações errôneas.

Com relação aos capítulos da cartilha, os títulos foram escritos na fonte Cooper Black, tamanho 54 para a capa, elaboração e sumário; tamanho 33 para o conteúdo textual, referências e anotações, prevalecendo nos títulos dos capítulos a cor preta. Enquanto o conteúdo textual foi escrito utilizando a fonte Times New Roman, cor preta, tamanho 24. As informações textuais foram apresentadas preferencialmente, de forma justificada e com espaçamento entre as linhas de $1,5 \mathrm{~cm}$. Procurando sempre evitar o uso de palavras em caixa alta e em itálico, pois o texto se torna incompreensível e sem foco quando é utilizado em uma mesma página, vários tamanhos e tipos de letra (MOREIRA; NOBREGA; SILVA, 2003).

Quanto as informações textuais, estas foram organizadas de maneira didática, iniciando com a definição de hipertensão e, em seguida descrevendo as causas da doença, os fatores de risco, os sintomas da hipertensão, o efeito da hipertensão no organismo, como controlar a pressão alta, o que deve ser evitado e preferido, finalizando com dicas 
saudáveis. Na parte textual, procurou-se utilizar frases de extensão curta, pois de acordo com Oliveira e colaboradores (2007), frases longas reduzem a velocidade da leitura e podem levar à desistência desse ato.

Ademais, o Ministério da Saúde recomenda que na produção textual de um material didático, a linguagem seja clara, objetiva e coloquial, adequada às características da clientela, pois isso permite que a leitura seja leve e de fácil compreensão pelo públicoalvo (BRASIL, 2005).

Quanto às ilustrações, essas foram utilizadas com o intuito de ajudar na explicação ou enfatizar ideias abordadas no texto. Para tal, nas imagens colocou-se uma idosa, mostrando expressões faciais relacionadas com as informações textuais. Para Martins et al. (2019), a estratégia de projetar a imagem do público-alvo na cartilha, faz com que o leitor se identifique com o personagem e se imagine vivenciando os eventos.

Segundo Doak e colaboradores (1996) e Moreira, Nóbrega e Silva (2003), as ilustrações ajudam a fixar o conteúdo e contribuem para que as pessoas com pouca escolaridade se sintam motivadas a ler.

Com relação à diagramação, etapa que corresponde à organização, formatação e construção da cartilha, foram utilizados o programa Corel Draw Essentials para desenhar/ilustrar, o programa Adobe Photoshop para colorir as figuras e o Adobe Indesign®. para a diagramação e configuração das páginas.

A cartilha foi desenvolvida em folha A4 $(210$ x 297mm) com layout orientado em forma de retrato, sendo que esta foi construída para ser impressa no tamanho $10 \times 14 \mathrm{~cm}$, pois nessas dimensões a cartilha caberá facilmente no lugar que o leitor desejar e pode ser transportada com facilidade.

Adotou-se a cor bege claro para o fundo da página da cartilha em busca de um aspecto de leveza para a leitura, ainda neste fundo foram implementadas bordas de cor vermelha no final de cada página, enaltecendo a cor simbólica das doenças do coração. Segundo Moreira e colaboradores (2003) e Amaral (2020), o fundo claro da cartilha não cansa a vista e facilita a leitura.

Assim, a versão final da cartilha foi intitulada de "Prevenção e Controle da Hipertensão Arterial”, sendo composta por 16 páginas, sendo 11 referentes ao conteúdo textual.

A seguir é descrita a composição da cartilha: 
A figura 1 correspondeu à capa, contendo o título e ano da publicação da cartilha, enquanto a figura 2 é referente à contracapa, na qual consta o nome do autor e do ilustrador do material educativo, bem como da responsável pela orientação da confecção da cartilha. No sumário (figura 3) consta todos os capítulos da cartilha com respectiva página.

Figuras 1, 2 e 3 - Capa, elaboração da cartilha e sumário.

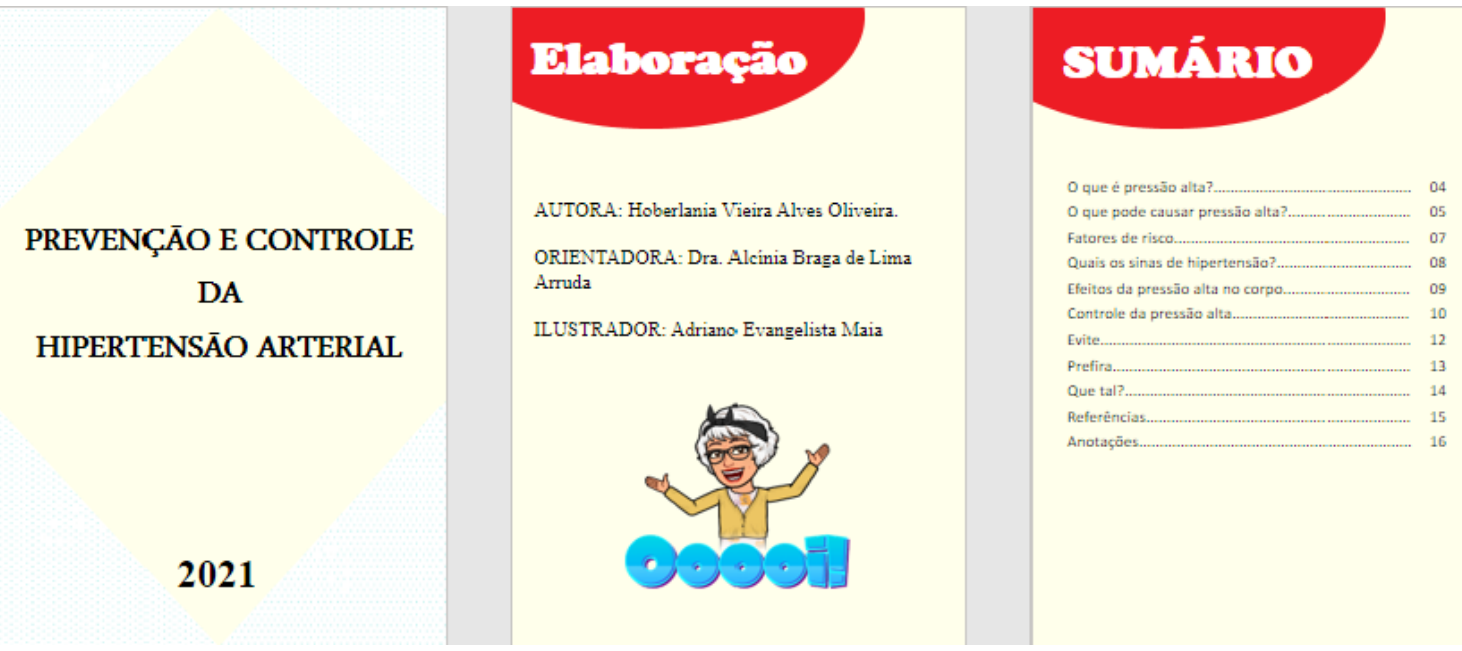

Fonte: Elaboradas pelos autores da pesquisa.

As figuras correspondentes às páginas 4,5 e 6 constam perguntas sobre o que é pressão alta e o que pode causar a pressão alta, as quais foram respondidas de maneira breve e em tópicos, com respectivas ilustrações para reforçar o aprendizado.

Figuras 4, 5 e 6 - Conteúdo textual (o que é pressão alta e o que pode causar a pressão alta).
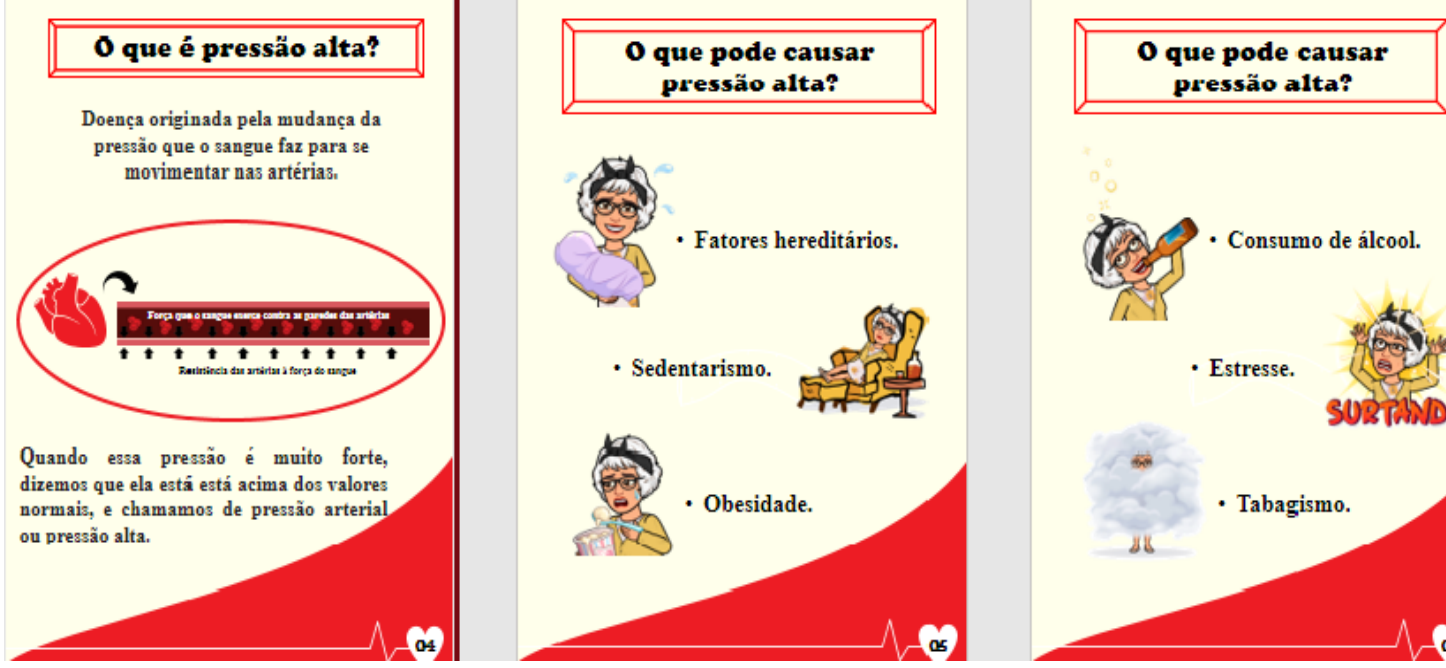

Fonte: Elaboradas pelos autores da pesquisa. 
A figura 7 descreve os fatores de risco para desenvolver a hipertensão. Enquanto as figuras 8 e 9, se referem aos sintomas da hipertensão e os efeitos da pressão alta no corpo, respectivamente.

Figuras 7, 8 e 9 - Conteúdo textual (Fatores de risco, Quais os sintomas da hipertensão, Efeitos da pressão alta no corpo)
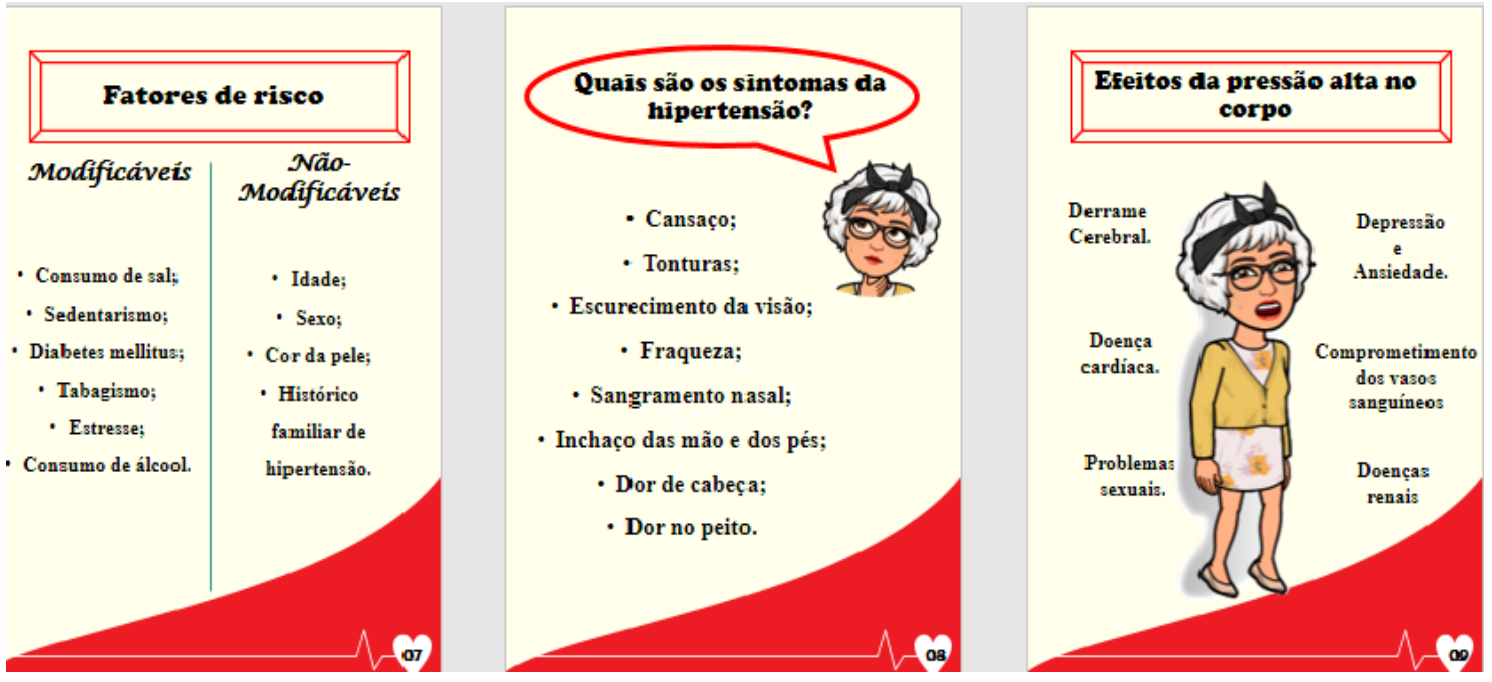

Fonte: Elaboradas pelos autores da pesquisa.

As figuras 10 e 11 mostram como as idosas podem controlar a pressão arterial. Enquanto que a figura 12 mostra o que deve ser evitado para a pessoa prevenir ou controlar a hipertensão.

Figuras 10,11 e 12 - Conteúdo textual (Controle da pressão e Evite)
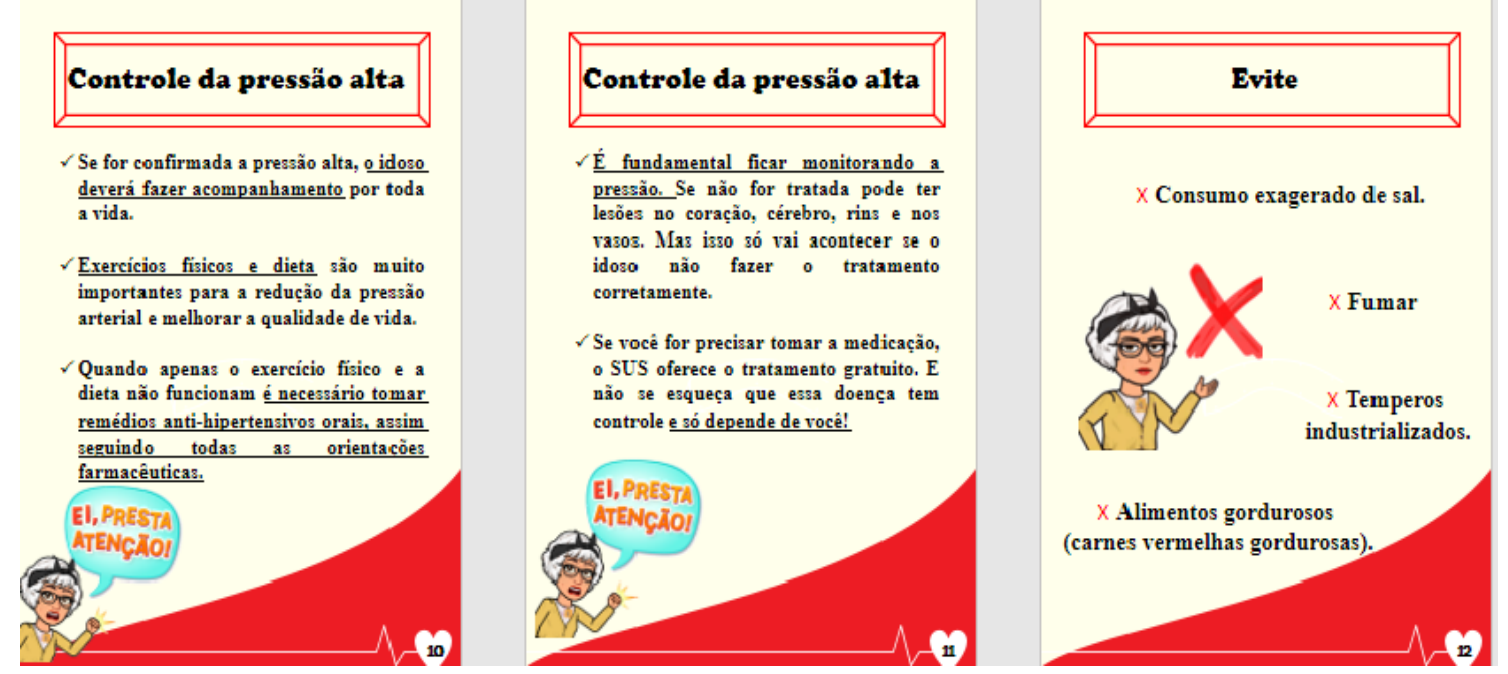

Fonte: Elaboradas pelos autores da pesquisa. 
As figuras 13 e 14 mostram o que as idosas devem preferir na sua alimentação e as atividades que essas devem fazer para prevenir ou controlar a hipertensão, respectivamente. A figura 15, por sua vez, consta as referências bibliográgficas utilizadas para a confecção da cartilha.

Figuras 13, 14 e 15 - Conteúdo textual (Prefira e Que tal) e Referências

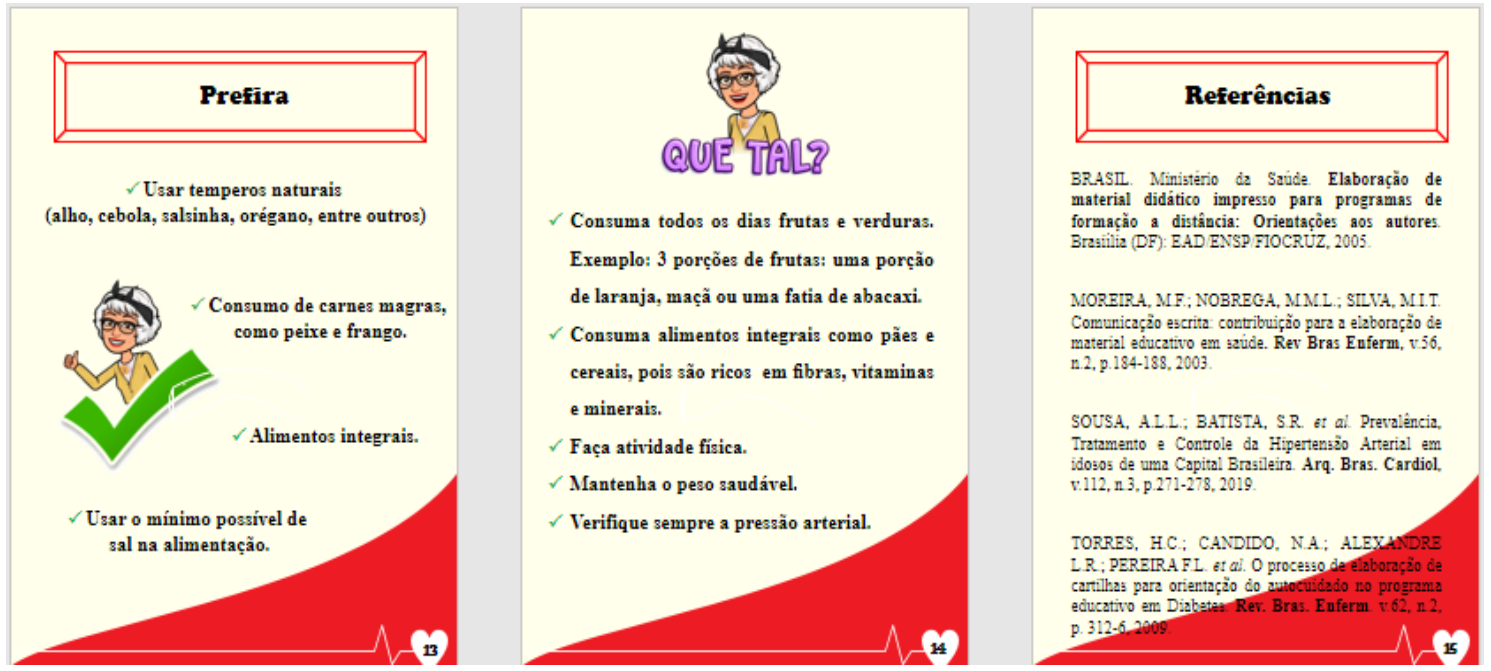

Fonte: Elaboradas pelos autores da pesquisa.

A figura 16 é referente a última página da cartilha, destinada à identificação da idosa e consta uma tabela para anotar os valores da pressão arterial, com o intuito de monitorar e estimular o autocuidado.

Figura 16 - Anotações

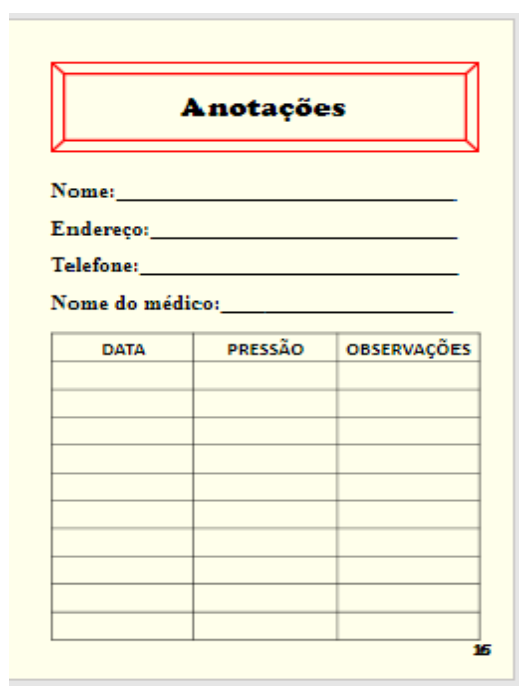

Fonte: Elaboradas pelos autores da pesquisa. 


\section{CONSIDERAÇÕES FINAIS}

$\mathrm{Na}$ confecção da cartilha utilizou-se todas as sugestões sugeridas pela literatura especializada, conseguiu-se assim, elaborar uma ferramenta didática simples, de fácil entendimento e com informações importantes que permitem o reconhecimento da hipertensão arterial, seus sintomas, prevenção e controle.

No entanto, a cartilha ainda precisa ser submetida à validação com a participação de juízes especialistas na área. A validação da cartilha é relevante, tendo em vista que as colaborações oriundas deste processo vão aprimorar a qualidade do material e permitir que esse possa ser utilizado com as idosas, seus familiares e cuidadores.

\section{REFERÊNCIAS}

AMARAL, A. Design de informação como ferramenta para a elaboração de uma cartilha com exercícios de Yoga para redução da ansiedade em idosos. 2020. $180 \mathrm{f}$. Trabalho de Conclusão de Curso. Bacharel do Curso de Design. Universidade do Sul de Santa Catarina. Florianópolis, 2020.

AMORIM, R. C.; LIMA, M. S. F.; JESUS, I. P. et al. Metodologia do processo de elaboração de cartilha informativa para orientar feirantes quanto à prevenção do novo coronavírus e da Covid-19. Raízes e rumos, v.8, n.1, p. 131-150, 2020.

BARROSO, W.K.S. et al. Diretrizes Brasileiras de Hipertensão Arterial - 2020. Arq Bras Cardiol. v.116, n.3, p.516-658, 2021.

BRASIL. Ministério da Saúde. Elaboração de material didático impresso para programas de formação a distância: Orientações aos autores. Brasiília (DF): EAD/ENSP/FIOCRUZ, 2005

BRASIL. Ministério da Saúde. Secretaria de Atenção à Saúde. Departamento de Atenção Básica. Informe da Atenção Básica n. 51, ano IX, mar/abr. 2009.

CASTRO, A.N.P.; LIMA JUNIOR, E.M. Desenvolvimento e validação de cartilha para pacientes vítimas de queimaduras. Rev. Bras. Queimaduras. v.13, n.2, p.103, 2014.

DANTAS, E.H.M.; SANTOS, C. A.S. Aspectos biopsicossociais do envelhecimento e a prevenção de quedas na terceira idade. organizadores Estélio Henrique Martin Dantas, César Augusto de Souza Santos. - Joaçaba: Editora Unoesc, 2017. 330 p.

DOAK, C.C.; DOAK, L.G.; ROOT, J.H. Teaching patients with low literacys kills. $2^{\mathrm{a}}$ ed. Philadelphia: J. B. Lippincott, 1996.

DUTRA, C.M.; COELHO, M.J. Implante de valva mitral mecânica: reflexões para cuidar e os cuidados de clientes após a alta hospitalar. Esc. Anna Nery. v.10, n.2, p.309-315, 2006. 
ECHER, I. C. Elaboração de manuais de orientação para o cuidado em saúde. Rev. Latino-am. Enfermagem. v.13, n.5, p. 754-757, 2005.

BORGES, E. et al. O processo de envelhecimento: as principais alterações que acontecem com o idoso com o passar dos anos. Rev. Cientifica Internacional. v.1, n.7, p. 106-132, 2012.

FERREIRA, O.G.L. et al. Envelhecimento ativo e sua relação com à independência funcional. Texto contexto Enferm, v.21, n.3, p. 513-8, 2012.

GALDINO, Y.L.S. Construção e validação de cartilha educativa para o autocuidado com os pés de pessoas com diabetes. 2014. 88f. Dissertação (Mestrado Acadêmico em Cuidados Clínicos em Enfermagem e Saúde). Centro de Ciências da Saúde da Universidade Estadual do Ceará, Fortaleza, 2014.

GOZZO, T.O. et al. Informações para elaboração de um manual educativo destinado a mulheres com câncer de mama. Revista de Enfermagem, v.16, n.2, p.306-311, 2012.

Instituto Brasileiro de Geografia e Estatística. IBGE. Pesquisa Nacional de Saúde 2013. Percepção do Estado de Saúde, Estilos de Vida e Doenças Crônicas. Rio de Janeiro, 2013.

\section{LIMA, A. C. M. A. C. C. Construção e validação de cartilha educativa para} prevenção da transmissão vertical pelo HIV. 2014. 136f. Dissertação (Mestrado) Faculdade de Farmácia, Odontologia e Enfermagem, Universidade Federal do Ceará, Fortaleza, 2014.

LIMA, C.N. Elaboração de uma cartilha educativa sobre a prevenção e controle do Diabetes, para ser utilizada com os idosos, seus familiares e cuidadores. 2020. 49f. Monografia (Graduação) - Departamento de Análises Clínicas e Toxicológicas, Faculdade de Farmácia. Universidade Federal do Ceará, Fortaleza, 2020.

MALTA, D.C.; BERNAL, R.T.I. et al. Doenças crônicas não transmissíveis e a utilização de serviços de saúde: análise da Pesquisa Nacional de Saúde no Brasil. Rev. Saude Publica 2017; 51(Supl. 1):4s.

MARTINS, R.M.G.; DIAS, I.K.R. et al. Desenvolvimento de uma cartilha para a promoção do autocuidado na hanseníase. Rev. Enferm. UFPE online. v.13, p.e239873, 2019.

MOREIRA, M.F.; NOBREGA, M.M.L.; SILVA, M.I.T. Comunicação escrita: contribuição para a elaboração de material educativo em saúde. Rev Bras Enferm, v.56, n.2, p.184-188, 2003.

MOURA, A.A.G.; CARVALHO, E.F.; SILVA, N.J.C. Repercussão das doenças crônicas não-transmissíveis na concessão de benefícios pela previdência social. Cien Saude Colet v.12, n.6, p.1661-1672, 2007. 
OLIVEIRA, V.L.B.; LANDIM, F.L.P. et al. Modelo explicativo popular e profissional das mensagens de cartazes utilizados nas campanhas de saúde. Texto Contexto Enferm, v.16, n.2, p.287-293, 2007.

OLIVEIRA, S.C.; LOPES, M.V.O.; FERNANDES, A.F.C. Construção e validação de cartilha educativa para alimentação saudável durante a gravidez. Rev Lat Am Enfermagem. v. 22, n.4, p.611-20, 2014.

PEREIRA, R. J.; COTTA, R. M. M.; FRANCESCHINI, S. C. C.; PRIORE, S. E. Características da saúde do idoso brasileiro. Rev. Med. Minas Gerais, v.19, n.1, pp.4450, 2009.

REBERTE, L.M. Celebrando a vida: Construção de uma cartilha para a promoção da saúde da gestante. 2008. Dissertação (Mestrado em Enfermagem). Escola de Enfermagem, Universidade de São Paulo, São Paulo, 2008.

SBC - Sociedade Brasileira de Cardiologia. VI Diretrizes Brasileiras de Hipertensão Arterial. Rev. Bras. Hipertens. v.13 n.4, p.256-312, 2010.

SOUSA, A.L.L.; BATISTA, S.R. et al. Prevalência, Tratamento e Controle da Hipertensão Arterial em idosos de uma Capital Brasileira. Arq. Bras. Cardiol, v.112, n.3, p.271-278, 2019.

TORRES, H.C.; CANDIDO, N.A.; ALEXANDRE L.R.; PEREIRA F.L. et al. O processo de elaboração de cartilhas para orientação do autocuidado no programa educativo em Diabetes. Rev. Bras. Enferm. v.62, n.2, p. 312-6, 2009.

VERAS, R.P. Prevenção de doenças em idosos: os equívocos dos atuais modelos. Cad. Saúde Pública. v.28, n.10, p.1834-1840, 2012.

VERAS, R.P.; OLIVEIRA, M. Envelhecer no Brasil: a construção de um modelo de cuidado. Ciênc. Saúde Colet, v. 23, n. 6, p. 1929 - 1936, 2018. 